\title{
Estudio cualitativo sobre factores sociales que influyen en la inclusión social de personas con sordoceguera
}

\author{
Mónica Ruiz González¹; Pablo de la Rosa Gimeno²
}

Recibido: 20/11/2019; Revisado: 10/12/2019; Aceptado: 11/05/2020

Resumen. El artículo recoge el resultado de una investigación cualitativa que descubre la influencia de determinados factores sociales en la autonomía e inclusión social de las personas con sordoceguera. Se han seleccionado cuatro grandes ámbitos sociales para observar su relevancia en la vida de estas personas: la familia, la participación social, el acceso a recursos y el acceso a las tecnologías de la información y comunicación. Se realizaron inicialmente una serie de entrevistas a expertos en la atención social a las personas con sordoceguera (informantes clave) que permitieron orientar adecuadamente las once entrevistas en profundidad realizadas a personas con sordoceguera de distinto perfil: edad, sexo, estado civil y situación laboral. La información obtenida ha permitido establecer 18 factores que contribuyen positivamente (9) o negativamente (9) al desarrollo de la autonomía personal e inclusión social de estas personas. Entre los factores positivos se destacan: la participación social (especialmente la interacción con otras personas sordociegas), el apoyo personalizado y el acceso a las tecnologías de la información y comunicación. En sentido contrario, como factores obstaculizadores de esa autonomía e inclusión, se encuentran: la sobreprotección familiar, la falta de profesionales expertos en sordoceguera y la escasez de servicios de apoyo a las familias de las personas con sordoceguera.

Palabras clave: Autonomía personal; integración social; sordoceguera.

\section{[en] Qualitative study on social factors influencing the social inclusion of deaf-blind persons}

\begin{abstract}
The article reports the findings of qualitative research to identify the influence of certain social factors in the autonomy and social inclusion of deaf-blind persons. Four major social areas were selected in order to observe their significance in these people's lives: family, social participation, access to resources and access to information and communication technologies. First, a series of interviews was conducted with experts in social care for deafblind persons (key informants). This permitted the appropriate structuring of the eleven in-depth interviews carried out with deaf-blind persons who had various profiles in terms of age, sex, marital status and employment situation. The information collected facilitated the identification of 18 factors that contribute positively (9) or negatively (9) to the development of personal autonomy and social inclusion for these people. Notable positive factors included social participation (particularly interaction with other deaf-blind persons), personalised support and access to information and communication technologies. In contrast, among the factors that inhibited this autonomy and inclusion were overprotective family, a lack of professional experts in deaf-blindness and scarcity of support services for the families of deaf-blind persons.
\end{abstract}

Keywords: personal autonomy; social integration; deaf-blindness.

Sumario: Introducción. 1. Marco teórico. 2. Metodología. 3. Resultados y discusión. 3.1. Familia. 3.2 Participación social. 3.3 Diseño de recursos. Las tecnologías de la información y la comunicación. 4. Conclusiones. Referencias bibliográficas.

Cómo citar: Ruiz González, M.; De la Rosa Gimeno, P. (2021) Estudio cualitativo sobre factores sociales que influyen en la inclusión social de personas con sordoceguera. Cuadernos de Trabajo Social, 34(1), 223-234.

\footnotetext{
Universidad de Valladolid monicaruizglez@gmail.com

2 Universidad de Valladolid pablo.rosa@uva.es
} 


\section{Introducción}

Nada hay más importante y característico de las personas que la libertad. Y la libertad supone, ni más ni menos, que el ejercicio de la autonomía personal. Por eso no hay labor más honorable que ayudar a las personas a mantener e incrementar su autonomía personal y, de este modo, aumentar su libertad. A esto se dedican las trabajadoras/es sociales.

Este artículo es resultado de la colaboración de dos personas que se dedican profesionalmente al Trabajo Social desde perspectivas distintas y, a la vez, muy cercanas: la profesional y la académica. Ella y él, dos investigadores que creen en las personas y en sus capacidades de ser autónomas. Ella, trabajando en un Centro de Acción Social de la ciudad de Valladolid, tuvo la oportunidad de tomar contacto por primera vez con personas con sordoceguera. Este contacto cambiaría para siempre la forma de mirar la sordoceguera, ya que lejos de encontrar en estas personas un grupo homogéneo de individuos altamente dependientes, pudo descubrir a una variedad de personas con grandes potencialidades, dispuestas a esforzarse lo que fuera necesario para aprender a hacer las cosas por sí mismas, rechazando ser sustituidas. Esta experiencia permitió a los autores de este artículo mirar la discapacidad de otra manera y preguntarse por las razones que explicaban las importantes diferencias que, en cuanto a autonomía funcional, existían entre distintas personas con sordoceguera, pese a que todas ellas compartían la misma discapacidad.

Esas preguntas llevaron a plantear una investigación que sirvió de base para la tesis doctoral titulada Autonomía e inclusión de personas con sordoceguera, defendida en la Universidad de Valladolid.

Existe un consenso generalizado, a la hora de definir la sordoceguera, (deafblind) como una discapacidad única, consistente en la combinación de la deficiencia visual y la auditiva que produce grandes dificultades en lo que respecta al acceso a la información, la comunicación y la movilidad y, en definitiva, en su autonomía y en la incorporación social plena (Wolff y Kennedy, 1994).

Se trata de una discapacidad única: el tacto como principal canal de comunicación. Única puesto que esta condición está generada por la combinación de la pérdida visual y auditiva, haciendo que la persona que la padece no pueda utilizar ni la visión ni la audición para comunicarse, sino que tenga que recurrir a otro sentido escasamente utilizado en las relaciones sociales de la vida cotidiana: el sentido del tacto. Evidentemente esta forma de recepción, comunicación y expresión tiene importantes consecuencias en la vida de la persona, en su desarrollo y aprendizajes.

Resulta muy relevante para el futuro de las personas con sordoceguera el orden de aparición de los déficits. No es lo mismo ni tiene las mismas consecuencias que primero se produzca la deficiencia auditiva y posteriormente la visual que lo contrario. Este hecho es crucial, ya que determina el lenguaje que utilizará la persona para comunicarse (oral o de signos) y su forma relacionarse (Álvarez, 2004).

Por otro lado, como factores exógenos destacan la actitud de la familia, el entorno en el que se desarrolla la persona con sordoceguera, y la existencia o ausencia de recursos de apoyo. Estos son los factores sobre los que se centró la investigación, utilizando el enfoque social de la discapacidad; esto es: la discapacidad entendida como resultado de la combinación de la limitación sensorial con el entorno.

Ante la necesidad de acotar el objeto de estudio se optó por elegir a personas con sordoceguera que han presentado grave deficiencia auditiva desde el nacimiento (o antes de los dos años de edad) y han desarrollado más tardíamente la deficiencia visual (en su mayoría, afectadas por el por el síndrome de Usher tipo I) y que, por lo tanto, su sistema de comunicación ha sido la lengua de signos (primero en el aire y posteriormente apoyada), por lo que resulta primordial el sentido del tacto para estas personas.

La toma de contacto con estas personas permitió descubrir, por un lado, que son personas que tienen una alta capacidad de superación y, por el otro, que el nivel de autonomía entre unos y otros sujetos es muy variable, incluso cuando presentan el mismo grado de afectación sensorial. Ello justifica el interés de descubrir los factores que favorecen la autonomía de las personas con sordoceguera con el propósito de coadyuvar al establecimiento de estrategias eficaces de atención social. El descubrimiento de estos factores será a partir de su discurso.

Por último, se hace necesario precisar que, a causa de la escasez de estudios sobre la materia, así como, a la propia dificultad en el acceso a estas personas con sordoceguera (tanto 
para localizarles como para establecer comunicación con ellas ${ }^{3}$ ), esta investigación tuvo un carácter exploratorio y prospectivo. Debido a este carácter, los resultados que se obtuvieron no deben generalizarse, pero sí pueden servir para apuntar hacia dónde deben dirigirse los recursos de apoyo a estas personas: al acompañamiento en el aprendizaje y no a la sustitución en la tarea. Con esta investigación se comprueba que las personas con sordoceguera cuentan con grandes capacidades y de ello se desprende, como podrá comprobar el lector, una de las principales conclusiones alcanzadas: solo si valoramos las potencialidades de estas personas, conseguiremos dar una respuesta satisfactoria a sus necesidades y expectativas. Tal y como se verá más adelante, los resultados obtenidos obligan a sustituir esa imagen inventada de sujetos dependientes por la imagen real del colectivo: personas capaces, capacitadas y ansiosas por ser autónomas y protagonistas de sus vidas.

\section{Marco teórico}

El estudio pretendía conocer la influencia de factores sociales como la familia, la participación social, el acceso a recursos y a las tecnologías de la información y comunicación en la autonomía de las personas con sordoceguera. De esta manera se podrán establecer eficaces estrategias de apoyo y promoción social a estas personas.

El concepto de autonomía es controvertido, no está exento de polémica. Desde un punto de vista normativo se puede acudir a la Ley 39/2006, de 14 de diciembre, de Promoción de la Autonomía Personal y Atención a las personas en situación de dependencia donde la autonomía es considerada como "la capacidad de controlar, afrontar y tomar, por propia iniciativa, decisiones personales acerca de cómo vivir de acuerdo con las normas y preferencias propias, así como desarrollar las actividades básicas de la vida diaria" (p. 44142). Se trata de un concepto relativo (nadie es ni totalmente autónomo ni totalmente dependiente) que no es posible reducir a la ejecución de tareas básicas, puesto que la persona, para ser autóno- ma, necesita poder elegir y controlar su propia vida, independientemente del nivel al que logre acceder y/o mantener a lo largo de su vida (Illán y Molina, 2013).

Con frecuencia, en la literatura especializada, el concepto de autonomía se asocia al de dependencia y discapacidad. En la Clasificación Internacional del Funcionamiento, de la Discapacidad y de la Salud (CIF, 2001) la discapacidad (disablement) se considera como un fenómeno multidimensional, resultado de la interacción de las personas con su entorno físico y social. La discapacidad, en estos términos, se produce por la interacción de las características de la persona con los factores contextuales. Este modo de entender la discapacidad, que recibe el nombre de modelo social (Palacios, 2008), se caracteriza por desplazar el núcleo del problema del individuo para situarlo en el contexto social. De ello se deduce que las respuestas a las limitaciones en la autonomía no deben centrarse sólo en las personas con discapacidad sino también en la sociedad, de manera que ésta se organice para atender las necesidades de todos sus miembros. Se comprueba así que las políticas de promoción de la autonomía personal realmente son, o deben ser, políticas de igualdad de oportunidades, de no discriminación centradas en la eliminación de obstáculos que impidan la participación plena de las personas con discapacidad en la vida cotidiana (Palacios y Romañach, 2006).

Además del modelo social, esta investigación asume el enfoque de las capacidades desarrollado por Amartya Sen (2001) y Martha Nussbaum (2012). Este enfoque "concibe cada persona como un fin en sí misma y no se pregunta solamente por el bienestar total o medio, sino también por las oportunidades disponibles para cada ser humano" (Nussbaum, 2012, p.38), situando en el centro de la intervención a la persona, trabajando con sus propias capacidades con objeto de ampliar su autonomía y, con ella, su libertad. Como se señala al comienzo de este apartado, se han considerado de manera especial cuatro factores sociales muy relacionados con la autonomía y calidad de vida de las personas con sordoceguera: la familia, la participación social,

\footnotetext{
Si fue posible llevar a cabo este trabajo de investigación fue gracias al apoyo de las dos asociaciones de personas con sordoceguera en Castilla y León, ASOCyL y ASOCIDE, así como, al Centro Santa Ángela de la Cruz (único centro de referencia estatal para personas con sordoceguera). Estas asociaciones no solo facilitaron el acceso a estas personas, sino que proporcionaron los medios técnicos y humanos y dotan del conocimiento necesario a la entrevistadora para llevar a cabo con éxito las entrevistas en profundidad.
} 
el diseño y acceso a recursos y las ayudas técnicas de las tecnologías de la información y la comunicación.

La familia. La familia puede considerarse como el principal agente socializador de la persona y, por lo tanto, es determinante en el desarrollo físico y emocional del niño. Esta realidad no es diferente cuando el niño que llega a la familia presenta discapacidad. Sin embargo, la información y el apoyo que habitualmente reciben los padres sobre la discapacidad de su hijo es muy limitado (Fernandes, Bascovi-Assis y Fiamenghi, 2010). Frecuentemente las indicaciones que reciben no suelen proporcionar asesoramiento suficiente sobre la discapacidad ni tampoco sobre los recursos a los que pueden optar en su comunidad, dejando a la familia llena de ansiedad cuando tiene que hacer frente a la nueva situación (Martins, 2002; Rodrígues y Dupas, 2011). La presencia en el hogar de un niño con alguna discapacidad se convierte en un factor que altera la dinámica familiar. Además, es importante tener en cuenta la necesidad de atención temprana de los niños y las familias durante los primeros años de vida, puesto que es cuando "se sientan las bases del desarrollo comunicativo y de la adquisición del lenguaje, así como de la maduración de la percepción auditiva y de todas las capacidades y habilidades cognitivas que de ella derivan" (Jaúdenes y Patiño, 2003, p. 51).

Una de las respuestas inadecuadas más frecuentes de las familias a sus hijos con discapacidad es la sobreprotección. La sobreprotección puede entenderse como un extremo de cariño que impide el correcto desarrollo de la persona. Tal y como señala Vázquez (2007), se trata de una forma inadecuada de interpretar la afectividad, tratando de evitarles los mayores riesgos posibles, privándoles así de la oportunidad de enfrentarse a las dificultades que surgen en el desarrollo de la vida y limitando, por tanto, su desarrollo personal.

La participación. Para García Roca (2004) "la participación implica, por una parte, arraigo, y por otra, posibilidad de elegir entre opciones. Es tanto un elemento de la voluntad, que se activa de forma deliberada y autónoma, como una estructura que lo posibilita" (p. 9). Idea que se completa con la de Sartori (1993) que entiende la participación como "incorporarse activamente a algo que se siente como propio, que es verdaderamente mío, decidido y perseguido libremente. No es un formar parte inerte ni es un ser obligado a formar parte" (p.
79). La participación se constituye en un factor esencial para conseguir la autonomía de las personas con sordoceguera.

Un espacio esencial de participación es el ocio. Para conseguir realmente una buena calidad en el ocio de la persona con sordoceguera, hay que posibilitar que ejerzan su derecho de autodeterminación desde el diseño,

No consiste en programar y ofertarles, sino diseñar desde sus propios criterios y demandas para realmente cubrirlas satisfactoriamente. Sobre todo, en personas adultas, es importante trabajar y diseñar con ellos, atendiendo a su perspectiva y a su visión del mundo (Ardura, 2000, p. 89).

El diseño de recursos. La sordoceguera es una discapacidad específica y heterogénea, por lo que no bastan servicios genéricos ni iguales para todos. El diseño de recursos requiere partir de un análisis de necesidades y demandas de la persona con sordoceguera; es decir, se debe crear partiendo de lo que requiere y desea la persona, sin dar por supuesto lo que necesita. Tampoco debe diseñarse por equiparación con otro tipo de discapacidades, que pueden no tener nada en común con la sordoceguera. Una cuestión que afecta de manera importante al diseño de recursos es el escaso conocimiento técnico-profesional sobre la sordoceguera, repercutiendo evidentemente este hecho en la atención a las necesidades y demandas de las personas que la presentan. Las personas con sordoceguera, para poder acceder a bienes y servicios, necesitan una ayuda previa; necesitan determinados medios para ello que no siempre se proporcionan o no se hace de forma efectiva para la persona y su familia. Si bien es cierto que se habla, cada vez más, de igualdad en el acceso o accesibilidad para todos, en los países donde existen servicios accesibles, generalmente están centrados más en una accesibilidad física que sensorial.

Las ayudas técnicas y las tecnologías de la información y la comunicación. La importancia de estas tecnologías para las personas con discapacidad y para las personas con sordoceguera en particular puede justificarse por diversos motivos, pero, en lo que al objeto de estudio respecta, destaca particularmente el potencial de las nuevas tecnologías para "aumentar sus posibilidades de relacionarse con el entorno y mejorar de esta forma su calidad de vida, afectiva, personal, emocional, laboral 
y profesional" (Cabero, Fernández y Córdoba, 2003, pp.15-16). Cuando se diseñan, esta tecnología no suele considerar las necesidades y características de las personas con discapacidad, provocando

La falta de accesibilidad en las aplicaciones deja excluido del manejo de las TIC al colectivo de personas con diversidad funcional, lo que incrementa su dependencia de otras personas para la realización de cierto tipo de tareas, así como su aislamiento social (Pousada y Pintos, 2012 p. 31).

\section{Metodología}

La metodología debe ser elegida en base a los objetivos de investigación. Como se adelantó en la Introducción, la investigación utiliza una metodología de enfoque cualitativo con pretensiones exploratorias y prospectivas. El objetivo general es descubrir qué factores familiares, sociales y materiales/tecnológicos favorecen el incremento de la autonomía de las personas con sordoceguera. Ello exige asomarse a la vida de estas personas, conocer su autonomía y comprobar cómo han influido en ella los factores familiares, sociales y materiales/tecnológicos. Para ello se preparó un guión de entrevista, siguiendo el enfoque de calidad de vida. El enfoque de calidad de vida ha sido y sigue siendo muy utilizado en el estudio de la persona con discapacidad y se considera muy adecuado para esta investigación, ya que permite poner el acento en los aspectos cualitativos. El concepto de calidad de vida en este artículo se plantea como elemento heurístico, ya que es una "noción sensibilizadora que nos proporciona referencia y guía desde la perspectiva del individuo, centrada en las dimensiones centrales de una vida de calidad" (Schalock y Verdugo, 2003, p.32).

La asociación entre esos asuntos necesariamente la deben hacer sus protagonistas: las personas con sordoceguera. El mejor modo de conocer cómo estiman y valoran la contribución de esos factores a su calidad de vida es mediante la entrevista en profundidad. Es el mejor modo de comprender cómo las personas ven y juzgan las cosas que les acontecen (Blumer, 1969). Consideramos la entrevista en profundidad como el encuentro "cara a cara entre el entrevistador y los informantes, encuentros éstos dirigidos hacia la comprensión de las perspectivas que tienen los informantes respecto de sus vidas, experiencias o situaciones, tal como las expresan con sus propias palabras" (Taylor y Bogdan, 1994. p. 101).

Elegido el método, es preciso seleccionar adecuadamente a las personas a entrevistar. Siguiendo a Valles (2002), el interés en la selección de los entrevistados no está solo a quiénes y cuántos entrevistar sino en lo que el autor denomina los principios muestrales de esas decisiones. Esta cuestión tiene particular importancia en esta investigación, ya que el universo del que se partía era ciertamente reducido en número, y las posibilidades de acceso al mismo eran escasas; por lo tanto, atender a esta cuestión ha sido tarea prioritaria. No puede obviarse la dificultad de la propia ejecución de las entrevistas, dado que entrevistadora y entrevistados no se comunicaban a través del mismo sistema, siendo necesaria la participación de un intérprete y en alguna entrevista dos (como la llevada a cabo con los entrevistados A y B, que se realizó de forma simultánea). Si estas entrevistas han podido celebrarse ha sido gracias a la generosa colaboración de intérpretes y de las personas con sordoceguera, que han estado dispuestos a relatar/interpretar todas aquellas cuestiones que fueron planteadas, con el cansancio que ello implicaba para unos y otros. La actitud de las personas con sordoceguera en las entrevistas fue extraordinariamente positiva, mostrando agradecimiento por ser objeto de interés de una investigación.

El primer criterio tenido en cuenta para la selección de las personas entrevistadas es lo que Valles (2002) denomina competencia narrativa atribuida. Las personas con sordoceguera son capaces de hablar sobre su propia vida, de relatar aquello que les ayuda o les dificulta en su día a día.

Como se verá más adelante, estas entrevistas se han llevado a cabo en lugares elegidos por los propios entrevistados (algunas en su propia casa, lo cual es, en sí mismo, otra fuente de información, que complementa el discurso de la propia persona).

Todas las personas que han participado en este estudio son personas que han presentado la deficiencia auditiva muy tempranamente -siempre antes de los dos años- mientras que la deficiencia visual de forma más tardía (en su mayoría, las personas entrevistadas, presentan síndrome de Usher tipo I). Este hecho responde a que, como ya se ha mencionado, los aprendizajes de las personas con sordoceguera son 
diferentes, dependiendo de que su deficiencia sea congénita o adquirida o si presentaron la limitación auditiva antes que la visual, ya que de ello depende que se adquiera o no la lengua oral y los aprendizajes posteriores.

Tabla 1. Perfil de las personas sordociegas entrevistadas

\begin{tabular}{|l|l|l|l|l|l|l|}
\hline & Edad & Sexo & Estado civil & Convivencia & Hijos & Experiencia laboral \\
\hline A & 41 & Mujer & Casada & Vive con su esposo & No & 2 años \\
\hline B & 43 & Hombre & Casado & Vive con su esposa & No & 5 años \\
\hline C & 45 & Hombre & Soltero & Vive solo & No & Ninguna \\
\hline D & 82 & Mujer & Viuda & Vive sola & 2 & 34 años \\
\hline E & 50 & Hombre & Soltero & Vive con sus padres & No & 3 años \\
\hline F & 50 & Mujer & Separada & Vive con su pareja & No & Ninguna \\
\hline G & 84 & Mujer & Viuda & Vive con un hijo & 4 & Ninguna \\
\hline H & 78 & Hombre & Casado & Vive con su mujer & 3 & 37 años \\
\hline I & 73 & Mujer & Soltera & Vive con su madre y hermano & No & 4 años \\
\hline J & 68 & Mujer & Casada & Vive con su marido & 5 & 33 años \\
\hline K & 65 & Mujer & Casada & Vive con su marido & 2 & 4 años \\
\hline
\end{tabular}

Fuente: Elaboración propia

El trabajo de campo tuvo lugar entre enero y diciembre de 2015. Para localizar a los posibles entrevistados se contactó con las dos asociaciones de personas con sordoceguera de Castilla y León: la Asociación de sordociegos de Castilla y León (ASOCyL) y la Asociación de sordociegos de España (ASOCIDE), delegación de Castilla y León. En el estudio de campo, las entrevistas fueron realizadas por la misma persona, la autora de esta investigación, con el apoyo de tres intérpretes.

Tan importante como la selección de los entrevistados resulta ser la preparación de la entrevistadora sobre el objeto y los objetivos de la investigación. Dada la temática de investigación, se valoró que era necesario que la entrevistadora contara con determinados conocimientos sobre la atención a las personas con sordoceguera, de cara a poder realizar las entrevistas con estas personas de forma eficiente. Con este objetivo, el de conseguir una adecuada capacitación para realizar las entrevistas (así como el de elegir adecuadamente los propios instrumentos de investigación), se realizó, por un lado, una visita al único centro de referencia estatal para personas con sordoceguera: el centro "Santa Ángela de la Cruz", ubicado en la localidad sevillana de Salteras (la visita tuvo lugar el 14 de septiembre de 2014) y, por el otro, varias entrevistas a expertos (informantes clave) en sordoceguera. Se realizaron tres entrevistas a trabajadores de diferentes en- tidades, con distintas formaciones y dedicados desde hace años, a la atención a las personas con sordoceguera. Estas entrevistas, junto con la visita al centro citado, perseguían la familiarización con el mundo de las personas con sordoceguera y conocer qué factores sociales, según los expertos, tenían relación más intensa con la autonomía de las mismas.

\section{Resultados y discusión}

La investigación permitió detectar numerosos factores que favorecen/inhiben el desarrollo de la autonomía e inclusión de las personas con sordoceguera. A continuación, se exponen aquellos que resultaron ser más significativos y recurrentes. Para facilitar su presentación, estos factores se presentan agrupados en los cuatro principales ejes relacionados con la autonomía de las personas con sordoceguera: familia, participación social, diseño y acceso a recursos y; ayudas técnicas y tecnologías de la información y la comunicación.

\subsection{Familia}

Los principales factores que inhiben la autonomía de la persona con sordoceguera son: la escasez de servicios de apoyo para las familias de estas personas, la sobreprotección familiar, la incomprensión familiar y el bajo conoci- 
miento de la lengua de signos por parte de la familia de origen.

Los expertos reconocen la dificultad que tienen las familias para recibir información sobre cómo atender a la persona con sordoceguera, al no existir apenas servicios de atención para ello:

Lo primero que demandan las familias es información, no saben cómo deben actuar ante la sordoceguera de su hijo...sería necesario crear un servicio de información para las familias, de cara a que pudieran conocer y comprender la sordoceguera (experto 3 ).

Los profesionales entrevistados señalan la necesidad de dotar de recursos (ampliar), optimizar (establecer criterios eficientes y justos) y protocolizar (cuándo una familia tiene un hijo con sordoceguera, debe saber qué existe y cómo tiene que proceder); actualmente, todo ello supone un auténtico desconcierto.

Este hecho parece conectar con el siguiente factor detectado que inhibe la autonomía de la persona con sordoceguera: la sobreprotección familiar. La sobreprotección ha sido detectada tanto en las entrevistas con las propias personas como en las mantenidas con los expertos. Los expertos entrevistados señalaban que la actitud más frecuente de las familias es justamente la sobreprotección, tratando de tranquilizar a la persona con sordoceguera, haciéndole ver que siempre van a estar ellos (u otro familiar) para cubrir todas sus necesidades:

Las PCS [personas con sordoceguera] lo pasan muy mal cuando fallecen sus cuidadores porque hasta ese momento no han sido conscientes de cómo iban a tener que vivir a partir de ese momento. Los familiares buscan tranquilizarles diciéndoles que no tiene de qué preocuparse, pero ese momento termina llegando, ya que lo habitual es que los padres mueran antes que los hijos y cuando eso ocurre, la PCS no está preparada para continuar con su vida... (Experto 1)

La sobreprotección, según los expertos entrevistados, está relacionada con el nivel formativo de las familias: cuánto más bajo es su nivel formativo, mayor es la sobreprotección que ejercen sobre la persona con sordoceguera. No obstante, los expertos destacaban que, a medida que transcurría el tiempo de trabajo con la familia, ésta modificaba su actitud de sobreprotección por otra más abierta. Esto pudiera indicar que cuando la familia recibe apoyos y se reconocen otras habilidades para tratar a su hijo, deja de sobreproteger; es decir, quizás la sobreprotección pueda superarse con la capacitación de las familias.

Otro de los factores que ha aparecido de forma significativa ha sido el desconocimiento de la lengua de signos por parte de la familia de origen. Los expertos señalaban que la familia habitualmente no conoce la lengua de signos, se comunican con la persona con sordoceguera a través de un sistema de comunicación alternativo que ellos mismos acordaban, consistente fundamentalmente en frases sencillas y gestos convenidos entre las partes, lo cual limita mucho la comunicación. De las once personas con sordoceguera entrevistadas, solo tres manifestaron que su padre o madre conocía la lengua de signos.

Derivado probablemente de los factores anteriores existe, así mismo, un sentimiento de incomprensión percibido por la persona con sordoceguera por parte de su familia. Hasta tal punto la persona llega a sentirse incomprendida que, según los expertos, las relaciones familiares se llegan a convertir en caóticas. Ante situaciones muy comprometidas y delicadas, las personas con sordoceguera no quieren que su familia sepa lo que les está ocurriendo, prefieren que no conozcan sus decisiones porque creen que no van a entender los motivos que han tenido para adoptarlas.

Es preciso profundizar en el estudio de estos factores y especialmente en lo referido a las estrategias más efectivas que permitan ofrecer apoyo psicosocial temprano a las familias, de forma que se puedan neutralizar factores que se han demostrado tan negativos como la sobreprotección o la incomprensión familiar.

\subsection{Participación social}

En lo que respecta a la participación social, los resultados fueron particularmente sugerentes. Destacan los siguientes factores como favorecedores de la autonomía de la persona con sordoceguera: la participación social mejora la autoestima, favorece la inclusión y disminuye la percepción de barreras; la participación favorece la autodeterminación de esta persona (elección desde el diseño); la interrelación con otras personas con sordoceguera favorece el sentimiento de comprensión en la persona con sordoceguera y la identidad como colec- 
tivo; formar parte de la toma de decisiones en lo que se hace supone el empoderamiento de la persona.

De las informaciones obtenidas en las entrevistas se desprende que la participación social mejora la autoestima. Las personas con sordoceguera reconocen sentirse mejor desde que acuden a la asociación:

Yendo a ASOCyL me olvidé de todo, sinceramente, ya no me acuerdo de que no veo y que no oigo; todo estaba adaptado a nuestras necesidades... la comunicación, la información, el relacionarme con otras personas y bueno...me he echado a la espalda todo lo malo (Entrevistado J).

Tal y como señalaron los expertos, las entrevistas a las personas con sordoceguera confirmaron que su mayor demanda se centra en poder comunicar y en relacionarse, siendo ambas cuestiones cubiertas cuando acuden a la asociación:

¡Me encanta realizar actividades con otras PCS! ¡Me encanta ir a la asociación, nuestra asociación! (Entrevistado B).

$\mathrm{Si}$ no existiese la asociación viviríamos completamente aislados (Entrevistada A).

Otro de los factores que se ha detectado con el trabajo de campo es que la participación disminuye la percepción de barreras. Las personas con sordoceguera referían de forma recurrente que perciben menos barreras desde que acuden a la asociación:

ASOCyL me ha permitido conocer las noticias, utilizar los medios de comunicación, asisto a un curso de dibujo del centro cívico (dibujo cómic), estoy realizando talleres de manualidades, viajes,... sobre todo destaco que tengo ilusión y ganas de vivir, que tengo ganas de que llegue la próxima cita para ir. Aquí siento que puedo conseguir todo (Entrevistado B).

Un elemento destacable que ha aparecido de forma sistemática en las entrevistas ha sido que la participación favorece la autodeterminación de la persona con sordoceguera (la elección de las actividades se realiza desde el diseño):

En la asociación se hace lo que dicen los sordociegos (Entrevistado C).
Así mismo, se ha detectado que la interrelación con otras personas con sordoceguera favorece una identidad como colectivo y dicha identidad colectiva supone, a su vez, una mejora en su autonomía. Las personas con sordoceguera manifestaban, por un lado, que se reconocen en ese otro que vive una situación similar a la suya (Pizzorno, 1989) y al mismo tiempo, ese reconocimiento les reportaba bienestar, al sentir que hay otras personas que tienen los mismos problemas y preocupaciones y que esas inquietudes, a su vez, son distintas a las que viven los otros:

Tengo muchas ganas de venir siempre y me gusta las bromas que hacen, me gusta contarles mis problemas, mis alegrías también... (Entrevistado $\mathrm{H})$.

Otro de los factores detectados en torno a la participación es que el asociacionismo les permite desarrollar su empoderamiento personal. Acudir a la asociación les ha abierto a un mundo nuevo, son más conscientes de la realidad, de sus derechos:

Mi vida está aquí, podría prescindir de muchas cosas, pero no de la asociación (...) en la asociación se hace lo que dicen los sordociegos (Entrevistado C).

Desde que acudo a la asociación, exijo que me den información, porque ahora sé que tengo derecho a recibirla (Entrevistada $G$ ).

Los importantes beneficios que la participación social de las personas con sordoceguera tiene en su desarrollo personal y calidad de vida, junto a la escasa exigencia de inversión económica de las acciones orientadas al fomento de la participación social, justificaría que las estrategias de atención a las PCS establecieran en el fomento de la participación social uno de los principales objetivos.

\subsection{Diseño de recursos}

En cuanto al diseño de recursos, han sobresalido en importancia los siguientes factores: diseño no adaptado a las características específicas de la persona (no bastan adaptaciones genéricas, para todos igual); falta de atención a la diversidad funcional de la persona con sordoceguera (destaca la ausencia de adaptaciones en la etapa escolar); la expectativa de atención de la persona con sordoceguera se centra en 
el acompañamiento y el aprendizaje y no en la prestación directa; insuficiente disponibilidad de servicios y apoyos técnicos cuándo se necesitan.

Se ha podido comprobar que los recursos no están adaptados a sus características personales, impidiendo así su uso (por ejemplo, un servicio de ayuda a domicilio prestado por una trabajadora que no conoce la lengua de signos) o incluso, aún peor, resultando que la prestación del recurso sea contraproducente:

Me ofrecían actividades en las que no podía participar, yo no podía aprender braille, por ejemplo, entonces lo que hacía era agobiarme aún más (Entrevistado J).

Puede resultar paradójico que en el ámbito escolar esta falta de adaptación haya sido tan evidente, pero la inexistencia de apoyos en esta esfera ha sido reconocida por la inmensa mayoría de los entrevistados. Nueve de las once personas con sordoceguera manifestaron que no tuvieron ninguna adaptación en su etapa escolar, los aprendizajes consistían en enseñarles como si fueran personas sordas, en la mayor parte de los casos:

Allí ni siquiera aprendía a leer ni a escribir...mis iguales me abandonaban, yo era para ellos el pesado (Entrevistado C).

En lo que respecta a la atención, la persona con sordoceguera busca más el acompañamiento y el aprendizaje que la prestación directa, demanda que se le apoye para desarrollar sus capacidades y no que se le den servicios que las nieguen o anulen:

Yo quiero hacer todo aquello que sea capaz de hacer, pero hacerlo yo y si necesito que me enseñen, pues que en eso consista la ayuda, en enseñarme no en hacerlo por mí (Entrevistado E).

Frecuentemente la disponibilidad de servicios y apoyos técnicos parece insuficiente. No es excepcional, por ejemplo, que una persona con sordoceguera necesite un intérprete o mediador para realizar una determinada gestión y este servicio no esté disponible para la fecha solicitada. Previsiblemente, la disponibilidad sea aún más baja cuando la persona no esté asociada, ya que estos ser- vicios se prestan fundamentalmente desde el tercer sector:

¿De qué me sirve tener derecho a un intérprete si no siempre que lo necesito está disponible? Además, tengo que solicitarlo con mucha antelación y a veces surgen cosas y claro, lo necesito rápidamente (Entrevistada $\mathrm{F}$ ).

Una vez más la evidencia contribuye a cuestionar aquellos programas de atención que se diseñan para ayudar y apoyar a determinados colectivos afectados por algunas carencias o limitaciones sin contar ni directa ni indirectamente con las propias personas a las que el programa pretende beneficiar.

\subsection{Las tecnologías de la información y la comunicación}

Cuando las tecnologías de la información y la comunicación se adaptan a las necesidades específicas de las personas con sordoceguera permiten satisfacer una de las mayores demandas de estas personas: la comunicación, expresiva y receptiva, reportándoles autonomía y libertad:

Ya no podría vivir sin internet. He aprendido muchas cosas, muchas recetas que no conocía, por ejemplo. Puedo hablar con amigos, hacemos vídeos y así si necesitamos cambiar la hora a la que hemos quedado o cualquier otra cosa, podemos hacerlo. Antes era imposible (Entrevistada $\mathrm{A}$ ).

Por otro lado, lamentablemente los expertos señalaban el desconocimiento que tiene la persona con sordoceguera de los productos de apoyo existentes, condicionando su demanda. Ello confirma que el acceso a información sobre la tecnología de apoyo es imprescindible. Otro aspecto negativo es que estas tecnologías suelen tener un elevado coste económico que dificulta el acceso a las mismas.

La investigación pone en evidencia la necesidad de que los modelos y estrategias de atención a las personas con sordoceguera garanticen su acceso a las tecnologías de la información y la comunicación mediante ayudas económicas que faciliten su adquisición y de procesos de acompañamiento que permitan una adecuada formación y adaptación del recurso a las características de la persona que lo usa. 


\section{Conclusiones}

Tal y como se ha reflejado al inicio de este texto, el origen de este trabajo de investigación partió de un descubrimiento, de una actitud de extrañamiento frente a una realidad que se había imaginado de manera diferente. El acercamiento a la vida de las personas con sordoceguera ha permitido comprobar que lejos de encontrar a individuos altamente dependientes y aislados, descubrimos a personas con grandes potencialidades, deseosas de participar y de tomar decisiones, dispuestas a luchar lo necesario para adquirir las habilidades que les permitieran "hacer todo aquello que pudieran", en definitiva: para ser libres.

Ha sido una experiencia extraordinaria descubrir cómo el tacto podía ser vector de una comunicación plena, admirar cómo con sus manos podían conversar, reír, llorar, disfrutar de la música, etc.
Pensábamos que la mejor manera de descubrir qué factores sociales, familiares y tecnológicos influyen en la adquisición de la autonomía de las personas con sordoceguera era asomarse a su realidad desde su propia perspectiva. Y no nos hemos equivocado. Han sido las personas con sordoceguera quienes, con sus relatos, han dado respuesta a los interrogantes de investigación y han ofrecido una imagen real de sus necesidades y capacidades. Quienes se vean implicados en orientar los recursos y programas de atención a las personas con sordoceguera, deberían asumir que la ayuda debe adaptarse a las personas, debe ser la respuesta a lo que la persona desea (y necesita), haciendo que sea la persona la que determine los objetivos y procesos de la ayuda, sin sustituirla.

En la siguiente tabla se recogen los principales resultados de la investigación a modo de resumen conclusivo:

Tabla n ${ }^{\circ}$ : Factores que favorecen/inhiben la autonomía de las PCS

\section{FACTORES QUE FAVORECEN LA AUTONOMÍA}

Participación social (mejora la autoestima, favorece la inclusión y disminuye la percepción de barreras)

Interrelación con otras PCS (favorece el sentimiento de comprensión en la PCS y la identidad como colectivo)

Formar parte de la toma de decisiones en lo que se hace (empoderamiento de la persona)

Acceso a la información

Comunicación efectiva (mejora y desarrollo de habilidades y sistemas de comunicación)

Adaptación en función de las características de la persona (no bastan adaptaciones genéricas, para todos igual)

Manejo y uso de las TIC (favorecen la comunicación, receptiva y expresiva)

Importancia del sistema de comunicación de la familia de origen (el que conozcan y favorezcan el uso de la LS)

Conocimiento y uso del bastón guía (favorece el desplazamiento, incluso en condiciones adversas)

\section{FACTORES QUE INHIBEN LA AUTONOMÍA}

Sobreprotección familiar

Inadaptación de los lugares (particularmente, la falta de luminosidad)

Incomprensión familiar

Sentimiento de infravaloración

Falta de adaptación a las características de las PCS en la etapa escolar

Escaso conocimiento técnico-profesional y diagnósticos insuficientes que permitan el avance de tratamientos y la visibilidad de las necesidades

Insuficiencia de profesionales expertos dedicados a la sordoceguera

No disponibilidad de los servicios y apoyos técnicos cuándo se necesitan

Escasez de servicios de apoyo para las familias de las PCS (necesarios para la comprensión de la sordoceguera, capacitación para su adecuado tratamiento...)

Fuente: elaboración propia

No podemos finalizar este texto sin reseñar el pretendido carácter innovador que tuvo la investigación. Innovador por distintas razones. Por un lado, por el propio objeto de estudio. Como se ha comentado anteriormente, la sordoceguera ha recibido escasa atención por parte de las po- 
líticas sociales y de los profesionales de la intervención social. Por otro lado, por la elección de una metodología cualitativa, interesada más en la comprensión de la vida de las personas que en el número de casos a estudiar. Y, por último, y quizá más importante, por los sujetos investigados. Las personas con sordoceguera entrevistadas nunca habían mantenido conversaciones tan largas e intensas sobre su propia vida y menos aún con objeto de formar parte de una investigación. Las muestras de agradecimiento por habernos interesado por sus vidas han constituido un extraordinario y emocionante descubrimiento. A ellos va dedicado este artículo.

\section{Agradecimientos}

Gracias a las personas con sordoceguera que tan generosamente han querido formar parte de esta investigación. Nunca se podrá agradecer lo suficiente su participación. Gracias por compartir con entusiasmo vuestras experiencias, por hablar con sinceridad y total entrega sobre vuestros sentimientos, vuestros retos, vuestras familias, vuestra asociación...en definitiva, sobre vuestras vidas.

\section{Referencias bibliográficas}

Álvarez, D. (2004). La sordoceguera. Una discapacidad singular. En: P. Gómez y E. Romero (coords.), La sordoceguera. Un análisis multidisciplinar. (pp. 135-192). Madrid: ONCE.

Ardura, A.J. (2000). Sordoceguera: ocio y tiempo libre. En: M.L. Setién (coord.), Ocio, calidad de vida y discapacidad. (pp. 87-92), Bilbao: Universidad de Deusto.

Blumer, H. (1982). El interaccionismo simbólico. Perspectiva y método. Barcelona: Hora.

Cabero, J., Fernández, J.M. y Córdoba, J. (2003). Las TIC como elementos en la atención a la diversidad. En: J. Cabero, J. Córdoba y J.M. Fernández (coords.), Las TIC para la igualdad. Nuevas tecnologías y atención a la diversidad. (pp. 15-35). Sevilla: Eduforma.

Fernandes, A.M., Blascovi-Assis, S.M. y Fiamenghi, G.A. (2010). Impacto da noticia da síndrome de Down para os país; histórias de vida. Ciencia \& Saude Coletiva, 15, 444-451.

García Roca, J. (2004). Politicas y programas de participación social. Madrid: Síntesis.

Illán, N. y Molina, J. (2013). La Filosofía de Escuela de Vida. Sus aportes para la promoción de la vida independiente en el colectivo de personas con discapacidad intelectual. Revista Latinoamericana de Educación inclusiva, 7(1), 23-38.

Jarrold, K. (2014). European Deafblind Indicators. Bruselas: European Comission: European Deafblind Network (EDbN).

Jaúdenes, C. y Patiño, I. (2003) Incidencia y repercusiones de la hipoacusia en niños. Detección precoz de la hipoacusia en el recién nacido. En: Ministerio de Sanidad y Consumo (ed.), Libro blanco sobre hipoacusia. Detección precoz de la hipoacusia en recién nacidos. (pp. 13-24). Madrid: Ministerio de Sanidad y Consumo.

Martins, D.A. (2002). Cuidando do portador de Síndrome de Down e seu significante. Ciênc cuidad saúde, 1(1), 111-116.

Mercado, E. y González, D. (2019). Trabajo social, estigma y discapacidad. En: E.J. Gómez (coord.), Imagen, estigma y derechos humanos. Valencia: Tirant lo Blanch.

Nussbaum, M. (2012). Crear capacidades: propuesta para el desarrollo humano. Barcelona: Paidós.

OMS. (2001). Clasificación Internacional del Funcionamiento, de la Discapacidad y de la Salud. Madrid: Ministerio de Trabajo y Asuntos Sociales.

Palacios, A. y Romañach, J. (2006). El modelo de la diversidad: La Bioética y los Derechos Humanos como herramientas para alcanzar la plena dignidad en la diversidad funcional. La Coruña: Diversitas-AlES.

Palacios, A. y Romañach, J. (2008). El modelo de la diversidad: una nueva visión de la bioética desde la perspectiva de las personas con diversidad funcional (discapacidad). Intersticios: Revista Sociológica de Pensamiento Crítico, 2, 37-47.

Pizzorno, A. (1989). Algún otro tipo de alteridad: una crítica a las teorías de la elección racional. Madrid: Fundación Sistema.

Pousada, T. y Pintos, J. (2012). Proyecto In-TIC: Integración de las Tecnologías de la Información y las Comunicaciones en las personas con discapacidad. La Coruña: Centro IMEDIR - Universidad de Coruña. 
Rodrigues, M.D. y Dupas, G. (2011). Independence of children with Downsyndrome: the experiences of families. Revista Latino-Americana de Enfermagem, 19(4), 985-993.

Sartori, G. (1993). Democrazia: cosa è. Milano: Rizzoli.

Sen, A. (2001). La desigualdad económica. México: Fondo de Cultura Económica.

Schalock, R. y Verdugo, M.A. (2003). Calidad de vida: manual para profesionales de la educación, salud y servicios sociales. Madrid: Alianza.

Taylor, S.J. y Bodgan, R. (1994). Introducción a los métodos cualitativos de investigación. La búsqueda de significados. Barcelona: Paidós Ibérica.

Valles, M.S. (2002). Entrevistas cualitativas. Madrid: Centro de Investigaciones Sociológicas. Colección Cuadernos metodológicos, $\mathrm{n}^{\mathrm{O}} 32$.

Vázquez, M.A. (2012). La sobreprotección materna y su incidencia en el bajo rendimiento escolar en niños y niñas comprendidos entre las edades de 6 a 12 años. (Tesis doctoral inédita) Universidad de San Carlos de Guatemala, Guatemala.

Wolff, K. y Kennedy, C. (1994). Etiologies and Characteristics of Deaf-Blindness. Pittsburgh: University of Pittsburgh. 